\title{
Praktisches und Statistisches zur intrauterinen Behandlung.
}

Von

R. O Is ha usen.

Winckel's Monographie über, die Behandlung der Flexionen des Uterus mit intrauterinen Elevatoren" hat die Frage nach der Zweckmässigkeit dieser Behandlungsweise und der Art ihrer Ausführung von Neuem angeregt und bereits eine Erwiderung von gegnerischer ${ }^{-S e i t e ~ h e r v o r g e r u f e n ~(S p i e g e l b e r g ~ i n ~ d i e s e m ~}$ Archiv III. S. 159). Vielleicht werden andere Entgegnungen folgen. Es muss dies aber auch für die Anhänger genannter Behandlungsweise eine Aufforderung sein, mit ihren Erfahrungen nicht zurückzuhalten, vielmehr die durch eine noch zu kleine Zahl von Fällen gestiitzten Erfahrungen Winckel's zu bestätigen und eventuell zu corrigiren. Ich fühle mich meinerseits zu einer derartigen Publication um so mehr aufgefordert, als ich vor fünf Jahren (Monatsschr. f. Geburtsk. Bd. XXX) einige Erfahrungen schon veröffentlichte mit verhältnissmässig vielen Fällen, in welchen die Instrumente nachtheilige Folgen hatten. Meine seitdem gemachten, zahlreicheren Erfahrungen sind günstiger als die früheren, zumal insoweit es sich um Fälle handelt, in welchen man üble Wirkungen zu beklagen hatte.

Nachdem nun so eben Winckel alle bei dieser Behandlung in Frage kommenden Punkte ausführlich erörtert hat, wäre eine 
nochmalige, weitläufige Besprechung nicht zeitgemäss und werde ich mich darauf beschränken, über das Material, auf welches sich meine Angaben stützen, einen Ueberblick zu geben, die Resultate mitzutheilen, schliesslich kurz und ohne ausführliche Motivirung anzugeben, welche Normen sich mir aus meinen Erfahrungen ergeben haben für die Wahl der Fälle, die Wahl des Instrumentes und die Ausführungsweise der Behandlung,

Die Fälle, auf welche sich sämmtliche Angaben beziehen, stammen lediglich' aus der Privatpraxis. Es kamen mir in demselben Zeitraume gleichzeitig vor:

25 Anteversionen

128 Anteflexionen

50 Retroflexionen

94 Retroversionen

zusammen 297 Fälle.

Diejenigen Fälle, welche eine deutliche Axenbiegung des Organs darboten, sind natürlich nur zu den Flexionen gezählt, mochte eine Version dabei fehlen oder, wie gewöhnlich, vorhanden sein. Bei den Rückwärtslagerungen des Organes ist die Bestimmung darüber, ob man sie zu den Versionen oder Flexionen zählen soll, bekanntlich oft nicht leicht. Bestand bei nur minimaler Flexion eine starke Version, so rechnete der Fall zu den Versionen. War dagegen die Flexion ausgeprägt, so zählte der Fall als solche. Bei Complicationen von Retroversio mit Anteflexio, wie sie öfter beobachtet wurde, zählte nur die Flexion. Die wenigen Fälle von Lateralflexion oder reiner Lateralversion sind nicht berücksichtigt.

Von den oben aufgetührten 297 Fällen wurden intrauterin behandelt

$$
81=27,3 \%
$$

und zwar von 128 Anteflexionen $66=51,6 \%$

$$
\text { von } 50 \text { Retroflexionen } 13=26,0 \% \text {, }
$$

von 25 Anteversionen und 94 Retroversionen je 1 Fall. Es wurde mithin die mechanische Behandlung eingeleitet in $27,3 \%$ der Fälle; darunter aufgegeben $13 \mathrm{Mal}$, also fortgesetzt nur $68 \mathrm{Mal}$ d. h. in $22,9 \%$ der Fälle. Auch diese Procentzahl ist noch höher als die von Martin und Winckel angegebene Ziffer, und ich stehe nicht an, zu bekennen, sie ist grösser als sie sein sollte. Ich habe seitdem die Indication gegen früher etwas eingeschränkt, die Contraindication erweitert und behandle jetzt weniger als frïher mit intrauterinen Pessarien. Eine Aufstellung aus der letzten 
Zeit und der Zukunft meiner Praxis wird unzweifelhaft etwas andere procentische Verhältnisse der mechanischen Behandlung geben.

Zu diesen 81 Fällen treten noch 5 Fälle intrauteriner Behandlung bei Kranken, welche weder eine Version noch Flexion hatten. Auf diese 86 . Fälle, welche ich nach einander intrauterin behandelte, beziehen sich die folgenden Angaben.

Die intrauterinen Instrumente kamen zur Anwendung:

bei Frauen, welche geboren hatten, $25 \mathrm{Mal}$,

bei nulliparen Frauen

$55 \mathrm{Mal}$,

bei nulliparen Unverheiratheten

6 Mal.

Das summarische Resultat der Behandlung in sämmtlichen Fällen ist folgendes:

die Instrumente erregten perimetritische oder parametritische Processe

7 Mal,

wurden wegen Blutung oder Schmerzen wieder entfernt.

10 Mal,

wurden vertragen und die angefangene Kur wurde weitergeführt

$66 \mathrm{Mal}^{3}$ ),

fielen immer wieder heraus, weshalb die Behandlung aufgegeben wurde

3 Mal.

Sehen wir von diesen 3 Fällen $a b$, so wurde unter den übrigen nahezu im 5. Theile der Fälle das Instrument nicht vertragen; $10 \mathrm{Mal}$ war der Nachtheil jedoch ein schnell vorübergehender; 7 Mal ein erheblicher, da Entzündungen eintraten. Keiner aller dieser Fälle, also keiner von 86 , nahm übrigens einen tödtlichen Ausgang. Auch ist mir unter den übrigen Fällen, welche ich behandelt habe, kein Fall mit lethalem Ausgange vorgekommen.

Fragen wir nach dem Nutzen in jenen 66 günstigen Fällen, so kommt hier wesentlich zweierlei in Betracht: die Beseitigung der Beschwerden der Kranken und in vielen Fällen die Beseitigung der bestehenden Sterilität. Auch da, wo es sich um Hülfe für die letztere handelt, überwiegt doch für Arzt und Patientin gewöhnlich das Interesse für Beseitigung der meist gleichzeitig bestehenden Beschwerden. Für diesen Zweck ist eine Heilung in anatomischem Sinne (Heilung der Flexion) meistens nicht nöthig und

1) Nur von einer dieser Kranken, welche in ihre Heimath reiste und dort unter Aufsicht ihres Arztes das Instrument trug, habe ich nichts wieder erfahren und weiss ich nicht, wie lange sie das Instrument trug. 
wird auch nur selten erreicht. Leider bin ich ausser Stande, zu sagen, wie oft eine solche in jenen 66 Fällen eingetreten ist. Eine grosse Zahl der Patienten habe ich nach Beendigung der instrumentellen Kur nur noch ein einzelnes Mal gesehen und den schliesslichen Erfolg in anatomischer Hinsicht nicht controliren können. Eine vollständige Beseitigung der Flexion ist gewiss nicht häufig; dass sie vorkommt, habe ich aber doch an einer kleinen Zahl von Fällen gesehen und früher schon berichtet.

Die Besserung oder Beseitigung der Beschwerden setzt ganz gewiss einen Erfolg auch in anatomischer Beziehung voraus; doch braucht dies häufig nicht einmal eine deutliche Verminderung der Flexion, geschweige denn eine Beseitigung zu sein; es genügt vielmehr eine dauernde Erweiterung der im Kanale des Uterus stenosirten Stelle, um die Beschwerden erheblich zu lindern oder $\mathrm{zu}$ beseitigen. Dies gilt vorzugsweise von der hervortretendsten Beschwerde, der Dysmenorrhoe im engeren Sinne, den menstrualen Uterinkoliken. Mit der Besserung dieser pflegt diejenige der übrigen Beschwerden, der Neuralgien, wie der Migräne, Intercostal- und Lumbalneuralgieen und diejenige deș Allgemeinbefindens gleichen Schritt zu halten.

Ueber den Erfolg in dieser klinischen Hinsicht, worauf es natürlich allein ankommt, kann ich folgende Angaben machen: Von den 86 Fällen wurde nur $73 \mathrm{Mal}$ die Behandlung fortgeführt und konnte, weil 7 Mal Entzündung eintrat, nur in 66 Fällen von Erfolgen die Rede sein. Hiervon wurden in 17 Fällen nur zweifelhafte Erfolge für die Dauer erzielt, wenn gleich während der Dauer der Behandlung fast immer wesentliche Erleichterung. eintrat; in 18 Fällen war die Besserung eine erhebliche und nach längerer Zeit noch anhaltende; in 15 anderen Fällen eine noch so lange Zeit constatirte, dass eine radicale Beseitigung der Beschwerden angenommen werden konnte. Unter diesen 15 Kranken befanden sich 11, bei welchen die gleichzeitig bestehende Sterilität durch die Kur beseitigt wurde.

In 8 Fällen trat eine Besserung nicht ein; in 8 anderen endlich konnte der Erfolg nicht constatirt werden, weil die Patienten ausser Beobachtung kamen.

Die Dauer der Anwendung kann ich in 57 Fällen, in welcher die Kur nicht wegen irgend welcher krankhaften Erscheinungen unterbrochen zu werden brauchte, angeben. Sie betrug: 
in 8 Fällen nicht über 3 Wochen,



Die angegebenen Zeiten bezeichnen die Gesammtdauer der Anwendung in den einzelnen Fällen. Es ist aber zu bemerken, dass das Instrument, zumal in Fällen langdauernder Anwendung, nicht immer die ganze Zeit ohne Unterbrechung lag. In einzelnen Fällen wurde nach einmaliger Anwendung das absichtlich entfernte Instrument nochmals applicirt, weil der erwünschte Erfolg, sei es betreffs der Beschwerden, sei es betreffs der Sterilität; noch nicht eintreten war. Häufiger fand eine unfreiwillige Unterbrechung der Kur durch Herausfallen des Instrumentes statt. Die Zeiträume, in welchen das Instrument nicht im Uterus lag, sind selbstverständlich bei obigen Angaben nicht mit berechnet. In 6 Fällen, wo das Instrument über ein Jahr getragen wurde, betrug die Zeit resp.

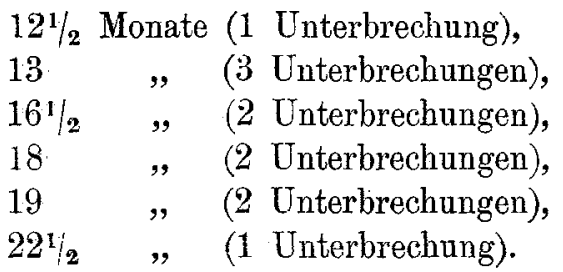

Die Erfahrungssätze, welche sich mir aus meinen Beobachtungen ergeben haben, möchte ich folgendermassen zusammenfassen:

Indicationen für die Anwendung:

Die Intrauterinpessarien sind vor Allem bei Behandlung von Flexionen zu. gebrauchen; für Versionen nur sehr selten; auch in einzelnen Fällen von Stenose des Uteruskanales ohne Flexion (klappenartige Ventile); endlich in einzelnen Fällen von Amenorrhoe.

Von den Flexionen eignen sich die Anteflexionen ungleich besser (gegen Winckel) als die Retroflexionen. Bei letzteren ist die Aussicht auf dauernden Erfolg wesentlich dadurch getrübt, dass bei dem Fortbestehen der Version und der Schlaftheit des Gebärmutterparenchyms die Flexion und Stenose des Kanales 
schnell wieder dieselben werden. Ausserdem hängen hier die Beschwerden eben so sehr und oft mehr von der incurabeln Version $a b$, als von der Stenose, welche das eigentliche Behandlungsobject für die intrauterinen Pessarien bilden soll. Auch ist die Anwendung der Instrumente bei Retroflexionen schwieriger und gefährlicher.

Die Indication zur Anwendung ist wesentlich zu entnehmen aus den deutlichen Erscheinungen der Stenose. Dieselbe wird sicherer erkannt aus der vorhandenen Dysmenorrhoe mit dem Charakter der mechanischen (kolikartige Schmerzen, im Beginne der Menstruation am stärksten, gewöhnlich mit baldigem Nachlass, bisweilen mit mehrtägigen Vorläufern), als aus der Sondirung, obgleich bei erheblicher Stenose auch die Sonde unzweifelhaft dieselbe nachweist.

Bei mangelnder Flexion und klappenartigem Ventil im Cervix kann die Sondirung unbehindert und doch der Austritt des menstrualen Blutes sehr erschwert und schmerzhaft sein. Vorhandene Sterilität, wo sie beseitigt werden soll, Neuralgieen und allgemeine Nervosität verstärken die Indication bei vorhandener Dysmenorrhoe. In ganz einzelnen Fällen können Blutungen die Indication abgeben. Ebenso sehr selten erhebliche Beschwerden, welche von einer Retroversio herrühren. Ungewöhnlich schlaffe Organe eignen sich wenig für die Behandlung. Adhäsionen, zumal wenig straffe, bilden keine entschiedene Contraindication, wenn sie auch die Aussicht auf Erfolg schmälern.

Contraindication geben natürlich ab alle peri- und parametritischen Complicationen. Die Schwierigkeit besteht hier in der Diagnose dieser Zustände, da wo sie einigermassen latent verlaufen. Von diagnostischer Wichtigkeit sind hier Schmerzen ausser der Zeit der Menstruation (von Uterinkoliken, wie sie in der Zwischenzeit bisweilen vorkommen, abgesehen), besonders anhaltende Schmerzen stechender Natur, welche durch Bewegungen und Erschütterungen vermehrt werden; ferner Schmerzhaftigkeit bei Bewegungen des Uterus oder ausgebreitete Empfindlichkeit des Organes bei Berührung von innen. (Eine, wenn auch erhebliche Empfindlichkeit blos an der Concavität der Knickungsstelle ist gewöhnlich eine neuralgische und giebt dann eine Contraindication nicht ab.)

Endlich ist zu beachten Druckempfindlichkeit am Abdomen, 
soweit nicht Neuralgieen der Lumbarnerven zu Grunde liegen, welche oft die excessivste Empfindlichkeit, bisweilen nur einseitig, bei blosser Berührung der Haut hervorrufen. Vorausgegangene Peritonitiden fordern um so mehr zur Vorsicht auf, wenn dieselben sich öfter und noch in letzter Zeit wiederholt haben oder gar nachweislich nach örtlichen Behandlungen eintraten.

Profuse Menstruation ist immer ein erschwerendes Moment für die Behandlung, aber fast nie von vornherein eine Contraindication; höchstens bei fehlgeschlagenem Behandlungsversuche. Das Gleiche gilt von intensiveren, chronischen Katarrhen des Uterus mit oder ohne Ulcus am Orificium externum.

\section{Wahl des Instrumentes. Applicationsweise.}

Als Material für die Instrumente genügt das Hartkautschuk jedenfalls allen Anforderungen. Es hält sich ungemein rein und wird nicht rauh oder angegriffen durch die Secrete. Von Horn, Knochen, Elfenbein lässt sich freilich dasselbe sagen. Holz und besonders Kork sind wegen der Imbibirbarkeit und damit bedingten Zersetzung der Secrete nicht zu empfehlen. Metallene Instrumente sind zu schwer und incrustiren sich leicht.

Das Instrument sei von möglicht einfacher Form, aus Stiel und Knopf bestehend, ohne Apparat zur Befestigung, wo es irgend angeht. Jede Art der Befestigung fixirt das Instrument in dem beweglichen Uterus und wirkt dadurch leichter reizend auf den letzteren.

Der scheibenförmige Knopf kann bei jungfräulichen Genitalien einen Durchmesser von $15-20 \mathrm{Mm}$. haben. Instrumente mit grösserem Knopfe lassen sich bei enger Vagina oft nicht, oder nur mit Schmerzen und stärkerer Reizung appliciren. Bei Personen, die geboren haben, ist meistens ein grösserer Knopf zu wählen, schon wegen der Gefahr des Verschwindens kleiner Knöpfe im Cervix (Haartmann); sodann auch, weil bei weiter schlaffer Vagina ein grösserer Knopf fester sitzt. Die kugelige Form der Knöpfe hat den Nachtheil leichteren Herausfallens. Besser ist die platte, oder bei kleinem Knopfe flach convex-concave, wie die Braunschen Instrumente sie haben. Bei grossem Knopfe scheint die ganz flache Scheibe, von 8-10 Mm. Dicke, dem Festsitzen des Instrumentes besonders günstig zu sein. 
Der Stiel soll möglichst dick gewählt werden bis zur Katheterdicke Nr. 6 (4 Mm.). Gelingt es das erste Mal nicht, einen leidlich dicken Stiel zu appliciren, so kann es von Vortheil sein, nach einigen Wochen ein dickeres Instrument einzulegen. Natïrlich muss der Stiel kürzer sein als die Höhle der Gebärmutter, durchschnittlich um 1,5-2,0 Cm. Nur in Fällen, wo nach der Application anhaltend ein Zwischenraum zwischen dem Orific. ext. uteri und dem der hinteren Vaginalwand aufliegenden Knopf sich findet, kann man bisweilen ein Instrument anwenden, welches nur wenig kürzer ist als die Höhle der Gebärmutter.

Der Knopf des Instrumentes soll die hintere Vaginalwand zum Stützpunkt haben, um nicht herauszufallen. Ist die Stellung der Gebärmutter normal, so stützt sich der Knopf in der bezeichneten Art wegen des Winkels, welchen die Axe des Uterus mit der des oberen Theiles der Vagina bildet. Besteht Anteversio, wie in fast allen Fällen von Anteflexio auch nach der Application des Instrumentes, so ist die Lage des Instrumentes um so gesicherter. Bei fehlender Version des Uterus genügt bisweilen ein einfacher Gummiring, welcher vor den Knopf des Instrumentes gelegt wird, um dasselbe festzuhalten. Der Gummiring hat nur den Zweck, den Knopf nach hinten zu drücken, wodurch mit ihm der Muttermund etwas nach hinten gedrängt, also eine Anteversionsstellung in geringem Grade bedingt wird.

Diese Art der Befestigung ist die schonendste und wird am leichtesten vertragen. Bei Retroflexionen fällt das Instrument, wenn es ohne weitere Vorsichtsmassregel eingelegt wird, fast immer sehr bald heraus. Ausgenommen hiervon sind Retroflexionen mit ziemlich starker Retro- und gleichzeitiger Lateralversion. Hier findet der Knopf bisweilen einen dauernden Stützpunkt an dem einen seitlichen vorderen Rande des Levator ani. Bei den meisten Retroflexionen ist es zweckmässig, zunächst das Organ an dem Knopfe des eingelegten Instrumentes aufzurichten, in Anteversionsstellung zu bringen, und, wenn es nicht in dieser verbleibt, mittels Gummiring oder ähnlicher Instrumente (zweckmässig gebogener Kupferring) in derselben zu fixiren. Rï̈ckt der Knopf immer wieder seitlich und nach vorn, so dass das Instrument herabrutscht, so kann man die Oeffnung des Gummiringes mit einem. engmaschigen Gitter fester Seide durchnähen und auf diesem den Knopf einen Stützpunkt finden lassen (ähnlich Winckel: Gummiring mit durchlöcherter Kautschukplatte). Die Controle 
über die Lage des Instrumentes ist leicht, da der Finger leicht um den Rand des dünnen Gummirings herumzubringen ist. Die viel dickeren, mit Krollhaar gepolsterten Pessarien sind aus diesem Grunde weniger zweckmässig. Auch ein hölzerner Teller mit abwärtsgerichtetem Stiele ist im Ganzen weniger passend.

Die sogenannten federnden Regulatoren, wie sie Martin und Winckel gebrauchen, habe ich mit Hildebrandt ganz aufgegeben. Sie reizen weit mehr, weil sie zu sehr fixiren, sind bei nicht sehr weiten Genitalien schwer zu appliciren und können nicht gut von einem Arzte, der das Instrument nicht kennt, entfernt werden.

Für die Art und Weise, wie das Instrument in den Uterus zu schieben ist, wird es wesentlich auf Uebung ankommen. Bei Retroflexionen ist die Einführung lediglich mit dem Finger wohl durchschnittlich das Beste. Bei Anteflexionen mittleren Grades mit nur mässiger Version ist dieselbe Art der Application bei einiger Uebung gewiss zu billigen. Bei einer starken, die Flexion complicirenden Anteversio und engen Genitalien ist die Einführung mittels biegsamer Sonde - welcher man für jeden Fall die geeignete Krümmung giebt - bei nur einiger Uebung, gewiss das Leichteste und Schonendste.

Die einmalige Sondirung unmittelbar vor der Application erleichtert diese stets, weil man dann genau die Richtung der Uterushöhle kennt und auch die Stenose momentan etwas weiter ist.

Nachtheilige Folgen der Instrumentalbehandlung.

Etwas Uterinkatarrh entsteht fast immer oder der schon vorhandene wird verschlimmert. Der Katarrh ist jedoch gewöhnlich nach wenigen Wochen für die Patienten kaum mehr merklich, hat stets einen gutartigen Charakter (schleimige oder seröse Secretion) und verliert sich nach. Beendigung der Kur auch dann, wenn dieselbe ein Jahr oder länger gedauert hatte, in kürzester Frist.

Profusere Menstruation ist im Beginne der Kur die gewöhnliche Folge derselben. Auch bei der zweiten Menstruation muss man auf eine etwas längere Dauer und stärkeren Blutverlust noch gefasst sein und in einer Anzahl Fälle dauert die Verstärkung der Menstruation so lange wie das Instrument liegt. War jedoch dieselbe nicht schon vorher ungewöhnlich stark, so pflegt der Blutverlust auch nicht während der Kur in nachtheiliger Weise auf- 
zutreten. In der Mehrzahl der Fälle ist von der zweiten Menstruation an die Blutung gar nicht oder sehr mässig verstärkt.

Blutabgang ausser der menstrualen Epoche oder eine sich sehr in die Länge ziehende, blutwässrige Absonderung nach der Menstruation deuten meistens auf eine Dislocation des Instrumentes, welche ausser durch die Defaecation gerade durch profuse Menstruation am Leichtesten bewirkt wird.

Wenn Spiegelberg (dieses Archiv III. S. 161) die Unschädlichkeit der intrauterinen Stifte in vielen Fällen durch eine Täuschung zu erklären sucht, indem er ,dieselben, wo sie längere Zeit getragen wurden, immer unter dem inneren Muttermunde, oder ...... unter der Knickungsstelle mit ihrer Spitze liegend fand", so muss ihm jeder mit der Sache Vertraute zugeben, dass dies bisweilen vorkommt. Aber es pflegen in solchen Fällen sehr bald unangenehme Folgen einzutreten - Schmerzen und anhaltend blutwässriger Abgang - welche auf die eingetretene Dislocirung des Instrumentes den Arzt aufmerksam machen, wenn dasselbe nicht, was ganz gewöhnlich ist, nachdem es mit seiner Spitze die stenosirte Stelle verlassen hat, auch aus der Vagina herausfällt. Dass es nach der genannten Dislocirung noch wochenlang in den Genitalien sich hält, gehört geradezu zu den Seltenheiten und kommt fast nur bei stark seitlicher Lage des Orific. uteri ext. vor. Ist aber eine solche Dislocirung eingetreten, so hat es für den, welchem die Maasse der Uterushöhle und des eingelegten Stiftes bekannt sind, keine Schwierigkeit, dieselbe zu erkennen.

Hieraus ergiebt sich, dass ich für meine Fälle mit Entschiedenheit, soweit ich nicht in einzelnen Fällen die gegentheilige Beobachtung machte und dann natürlich Correction eintreten liess, die dauernd richtige Lage der Instrumente behaupte. Zugleich bin ich aber auch der Ueberzeugung, dass Martin, Winckel, Hildebrandt, Hartmann, da sie so gut wie ich ihre Kranken controlirt haben werden, sich in der genannten Beziehung nicht getäuscht haben. Die obige Behauptung Spiegelberg's bleibt mir deshalb nach jeder Richtung hin unbegreiflich.

In seltenen Fällen ist die Application in den ersten Stunden von Uebelkeiten gefolgt, welche durch horizontale Lage meist schnell und für immer vorübergehen.

Eben so selten, etwa in jedem achten Falle auftretend, beobachtet man Uterinkoliken nach der Einlegung des Instrumentes, 
welche durch einige Gaben Morphium in ein bis zwei Tagen beseitigt werden. Wo eine ausgeprägte Hyperaesthesie der Genitalorgane bestand, an welcher Vulva, Vagina und Uterus Theil nehmen können, kann man Koliken am ersten erwarten. Gerade diese Kranken haben, nach Ueberwindung der ersten Tage, den eclatantesten Vortheil der Behandlung, weil ihre vorher bestehenden, oft sehr erheblichen Beschwerden in 8-14 Tagen meist ungemein gebessert sind.

Die nachtheiligste Folge, peri- und parametritische Affection, entsteht, wo sie eintritt, meistens in den ersten 14 Tagen; bisweilen später; in einzelnen Fällen mit einer intensiven Peritonitis; gewöhnlich schleichend. Spontane, nicht kolikartige Schmerzen, Druckempfindlichkeit bei äusserer und innerer Untersuchung, besonders bei Bewegungen des Uterus, Schmerzen beim Gehen der Patientin müssen auch bei noch vorhandener Fieberlosigkeit eine Aufforderung sein, das Instrument sofort zu entfernen.

\section{Dauer des Gebrauches.}

Die Dauer der Anwendung wähle man nicht zu kurz. In frischen Fällen - wenn z. B. die einzige vorausgegangene Geburt, wie öfters, mit Wahrscheinlichkeit als der Ausgangspunkt der Flexion bezeichnet werden kann und die Geburt erst. 1 oder $1 \frac{1}{2}$ Jahre her ist - wo man also noch auf Radicalheilung hoffen kann, ist das Instrument ein Jahr oder längere Zeit anzuwenden. Wo man nur palliative Erfolge hoffen kann, ist ebenfalls eine ausgedehntere Kur von nachhaltigerem Einflusse und um so nothwendiger, je intensiver und älter die Beschwerden sind.

\section{Resultate.}

Radicale Erfolge in anatomischem Sinne - Beseitigung der Flexion - sind selten, eine dauernde Erweiterung der vorhandenen Stenose und eine Verkürzung des verlängerten Uterus kommen öfter vor. Radicale Wirkung in Bezug auf die Beseitigung der Beschwerden tritt in der Minderzahl der Fälle auf; sehr häufig aber eine derartige Minderung derselben, dass die Patientin damit zufrieden ist. Der Erfolg für die Dysmenorrhoe ist schon bei der ersten Menstruation ein vollständiger, für die Hyperaesthesie der Genitalorgane meistens innerhalb acht Tagen zu constatiren; für die Kreuzschmerzen und Intercostalneuralgieen in wenigen Wochen; 
482 Olshausen, Praktisches und Statistisches zur intrauterinen Behandlung.

in etwas längerer Zeit erst für die Migräne. Damit bessert sich das Allgemeinbefinden, physisches und psychisches. Aehnliche Erfolge werden in den betreffenden Fällen durch keine andere Behandlung erzielt; für die Beseitigung der Dysmenorrhoe allerdings die fast gleichen Erfolge durch blosse Sondirung vor dem Eintritte der Menses. Doch hilft diese nur für ein einzelnes Mal. Selbst Monate lang fortgesetztes tägliches Sondiren hilft höchstens für zwei oder drei Menstruationen.

Aus diesen Gründen ist die Behandlung mit Intrauterininstrumenten unersetzbar und unter sorgfältiger Auswahl der Fälle nicht nur erlaubt, sondern indicirt. Die Schwierigkeit in der Auswahl der Fälle setzt eine genaue Kenntniss der betreffenden Affectionen und Gewandtheit in gynäkologischen Explorationen voraus. Dies und die Nothwendigkeit der Uebung in der oft schwierigen Application lassen es rathsam erscheinen, dass derartige Behandlungsweisen vor der Hand nur von Denjenigen ausgeführt werden, welche die obigen Bedingungen erfüllen können, d. h. sich mit Gynäkologie viel beschäftigt haben. Auch in der Hand Solcher schwinden die Gefahren der Behandlungsweise nicht ganz. Doch hat dieselbe dies gemeinsam mit zahllosen chirurgischen Operationen, welche nicht wegen lebensgefährlicher Affectionen unternommen werden; ebenso mit zahllosen anderen Kuren, welche am unrechten Orte unternommen, dem Kranken nichts nützen, sondern schaden. So wenig wie die meisten chirurgischen Operationen, welche nicht zur Lebensrettung unternommen werden, ist die intrauterine $\mathrm{Be}$ handlung der Gefahren wegen völlig zu verwerfen. 